\title{
Molecular cloning of the myo-inositol oxygenase gene from the kidney of baboons
}

\author{
RAFAEL GONZÁLEZ-ÁLVAREZ1 ${ }^{1}$, DIANA CRISTINA PÉREZ-IBAVE², MARÍA LOURDES GARZA-RODRÍGUEZ³, \\ ÁNGEL LUGO-TRAMPE ${ }^{4}$, IVÁN DELGADO-ENCISO ${ }^{5}$, MARÍA ELIZABETH TEJERO-BARRERA ${ }^{6}$, \\ LAURA ELIA MARTÍNEZ-DE-VILLARREAL ${ }^{7}$, RAQUEL GARZA-GUAJARDO ${ }^{8}$, \\ MARÍA MARISELA SÁNCHEZ-CHAPARRO ${ }^{7}$, GABRIEL RUIZ-AYMA ${ }^{9}$, \\ ORALIA BARBOZA-QUINTANA ${ }^{8}$, HUGO ALBERTO BARRERA-SALDAÑA ${ }^{3}$, \\ MARÍA DEL REFUGIO ROCHA-PIZAÑA ${ }^{10}$ and IRÁM PABLO RODRÍGUEZ-SÁNCHEZ ${ }^{7}$
}

\begin{abstract}
${ }^{1}$ Facultad de Medicina, Universidad Autónoma de Guadalajara, Zapopan, Jalisco 45129; ${ }^{2}$ Servicio de Oncología, Universidad Autónoma de Nuevo León, Centro Universitario Contra el Cáncer, Hospital Universitario 'Dr José Eleuterio González’; ${ }^{3}$ Departamento de Bioquímica y Medicina Molecular, Facultad de Medicina, Universidad Autónoma de Nuevo León, Monterrey, Nuevo León 64460; ${ }^{4}$ Centro Mesoamericano de Estudios en Salud Pública y Desastres (CEMESAD, Nodo Tapachula), Universidad Autónoma de Chiapas, Tuxtla Gutiérrez, Chiapas 29076; ${ }^{5}$ Facultad de Medicina, Universidad de Colima, Colima 28040; ${ }^{6}$ Instituto Nacional de Medicina Genómica (INMEGEN), Laboratorio Nutrigenética y Nutrigenómica, Tlalpan, Arenal Tepepan 14610; ${ }^{7}$ Departamento de Genética y Defectos Congénitos, Universidad Autónoma de Nuevo León, Centro Universitario Contra el Cáncer; ${ }^{8}$ Servicio de Anatomía Patológica y Citopatología, Universidad Autónoma de Nuevo León, Hospital Universitario ‘Dr José Eleuterio González’, Monterrey, Nuevo León 64460; ${ }^{9}$ Departamento de Ecología, Universidad Autónoma de Nuevo León, Facultad de Ciencias Biológicas, San Nicolás de los Garza, Nuevo León 66425; ${ }^{10}$ Centro de Biotecnología-FEMSA, Tecnológico de Monterrey Campus Monterrey, Monterrey, Nuevo León 64849, Mexico
\end{abstract}

Received January 3, 2017; Accepted February 8, 2017

DOI: $10.3892 /$ br.2017.973

\begin{abstract}
The enzyme myo-Inositol oxygenase (MIOX) is also termed ALDRL6. It is a kidney-specific member of the aldo-keto reductase family. MIOX catalyzes the first reaction involved in the myo-inositol metabolism signaling pathway and is fully expressed in mammalian tissues. MIOX catalyzes the oxidative cleavage of myo-Inositol and its epimer, D-chiro-Inositol to D-glucuronate. The dioxygen-dependent cleavage of the C6 and $\mathrm{C} 1$ bond in myo-Inositol is achieved by utilizing the $\mathrm{Fe}_{2+} / \mathrm{Fe}_{3+}$ binuclear iron center of MIOX. This enzyme has also been implicated in the complications of diabetes, including diabetic nephropathy. The MIOX gene was amplified with reverse transcription-polymerase chain reaction from baboon tissue samples, and the product
\end{abstract}

Correspondence to: Professor Irám Pablo Rodríguez-Sánchez, Departamento de Genética y Defectos Congénitos, Universidad Autónoma de Nuevo León, Centro Universitario Contra el Cáncer, Hospital Universitario 'Dr. José Eleuterio González', Avenida Gonzalitos s/n, Colonia Mitras Centro, Monterrey, Nuevo León 64460, Mexico

E-mail: iramrodriguez@gmail.com

Key words: animal models, gene expression, kidney, myo-inositol oxygenase, Old World monkey was cloned and sequenced. MIOX expression in the baboon kidney is described in the present study. The percentages of nucleotide and amino acid similarities between baboons and humans were 95 and $96 \%$, respectively. The MIOX protein of the baboon may be structurally identical to that of humans. Furthermore, the evolutionary changes, which have affected these sequences, have resulted from purifying forces.

\section{Introduction}

In mammalian cells, the only pathway for inositol breakdown is via myo-Inositol oxygenase (MIOX) (1). MIOX is a non-heme iron enzyme, which performs the conversion of myo-Inositol to glucuronic acid, the first committed step in myo-Inositol catabolism $(2,3)$. The reaction involves oxidative cleavage of the bond between $\mathrm{C} 6$ and $\mathrm{C} 1$ to produce $\mathrm{D}$-glucuronic acid. D-glucuronic acid is successively converted to L-gulonate, 3-keto-L-gulonate, L-xylulose, xylitol, D-xylulose and D-xylulose-5-phosphate, which then enters the pentose phosphate cycle (4). MIOX is predominantly expressed in the renal tubules, but is also observed at lower levels in extra-renal tissues, where diabetic complications are widely shown (3). Altered inositol metabolism is implicated in a number of diabetic complications, including cataracts, nephropathy, retinopathy and neuropathy. MIOX enzymatic activity is proportional to its serum glucose concentration (5). In addition, there is evidence that a polymorphism (rs761745) in the promoter region of the MIOX gene is associated with the progress of type 1 diabetes mellitus $(1,6)$. 
The structure of the MIOX gene has been characterized in humans and other mammals, including mice and rats, and MIOX sequences for cows, wild boars, dogs, orangutans, rhesus monkeys, common marmosets, bonobos, northern greater galagos, and Tasmanian devils are reported in GenBank $(2,4,7)$.

In humans, the MIOX gene is located on chromosome 22 in band 22q13.3, and consists of a DNA coding sequence (CDS) of 858 nucleotides (nt) split by 11 introns (exon 1,15 nt; exon $2,81 \mathrm{nt}$; exon $3,81 \mathrm{nt}$; exon 4,163 nt; exon 5,68 nt; exon 6,110 nt; exon 7,68 nt, exon 8,50 nt; exon 9,113 nt; and exon 10,109 nt). The open reading frame (ORF) encodes a polypeptide of 285 amino acid residues with a mass of $33.01 \mathrm{kDa}(8)$.

Although the function of the MIOX enzyme in human physiology has been determined, there are few MIOX-associated studies (8). Currently, there are gaps in the genomic information of MIOX in experimental animal models; thus, characterizing the physiological differences between humans and other MIOX proteins is difficult. An animal model closely associated with humans was selected in the current study. The current study describes the gene encoding MIOX in the baboon (Papio hamadryas). It is hypothesized that the baboon is an ideal animal model due to the fact that baboons spontaneously develop type 1 and 2 diabetes, which is considered to be a physiological research advantage.

\section{Materials and methods}

Animal specimens and nucleic acid isolation from biological samples. Samples from three male baboons were collected from the following frozen archived tissues: Testis, kidney, heart, omental fat, skeletal muscle, pancreas, mononuclear cells, liver and hypothalamus. The samples were collected according to ethical guidelines reviewed by the Institutional Animal Care and Use Committee of the Texas Institute of Biomedical Research (TIBR) (San Antonio, TX, USA). The animals had been housed at the Southwest National Primate Research Center at TIBR and were sacrificed under humanitarian conditions. All of the baboons were housed together and fed ad libitum on standard low-fat chow (15\% Monkey Diet, 8715; Harlan Teklad Labs, Houston, TX, USA).

Total RNA was isolated from the tissue samples using TRIzol reagent (Invitrogen; Thermo Fisher Scientific, Inc., Waltham, MA, USA), according to the manufacturer's instructions. The RNA samples were treated with DNaseI (Invitrogen; Thermo Fisher Scientific, Inc.) for $10 \mathrm{~min}$ at $37^{\circ} \mathrm{C}$ to remove any remainder of genomic DNA. The quality and integrity of the RNA were evaluated using standard spectrophotometric and electrophoretic methods, respectively.

Reverse transcription $(R T)$ and polymerase chain reaction (PCR) amplification. The RT reactions were developed with $1 \mu \mathrm{g}$ total RNA using random primers and a high-capacity cDNA RT kit (Applied Biosystems; Thermo Fisher Scientific, Inc.), according to the manufacturer's instructions. To amplify the baboon MIOX cDNA with PCR, 2X Taq PCR Master Mix (Qiagen, Inc., Valencia, CA, USA) was used, $5 \mu 1$ synthesized cDNA served as the template, and $10 \mathrm{pM}$ of each
Table I. Primates MIO-X sequences from NCBI GenBank.

Species (scientific name)

mRNA accession no.

Apes

$\begin{array}{ll}\text { Human (Homo sapiens) } & \text { NM_017584.5 } \\ \text { Pygmy chimpanzee (Pan paniscus) } & \text { XM_003811021.1 } \\ \text { Gorilla (Gorilla gorilla) } & \text { XM_004063692.1 } \\ \text { Sumatran orangutan (Pongoa belii) } & \text { NM_001131282.2 }\end{array}$

Old World monkeys

Hamadryas baboon (Papio hamadryas) KP299165

Olive baboon (Papioanubis)

XM_003905754.1

Gibbon (Noma scusleucogenys)

XM_003281467.1

Rhesus monkey (Macaca mulatta)

XM_001116334.2

Crab-eating macaque

XM 005566896.1

(Macaca fascicularis)

New World monkeys

Squirrel monkey

XM_003932782.1

(Saimiri boliviensis boliviensis)

Lemur (out-group)

Galago (Otolemur garnettii)

XM_003783084.1

Table II. Evolutionary forces that underlie the process of divergence in the MIO-X primate genes.

\begin{tabular}{lccc}
\hline Species & $\mathrm{dN}$ & $\mathrm{dS}$ & P-value $^{\mathrm{a}}$ \\
\hline Human & 0.0339 & 0.5298 & 0.0001 \\
Pygmy chimpanzee & 0.0347 & 0.5633 & 0.0001 \\
Gorilla & 0.0365 & 0.4986 & 0.0001 \\
Sumatran orangutan & 0.0348 & 0.4868 & 0.0001 \\
Hamadryas baboon & 0.0390 & 0.5404 & 0.0001 \\
Olive baboon & 0.0348 & 0.5344 & 0.0001 \\
Gibbon & 0.0330 & 0.4781 & 0.0001 \\
Rhesus monkey & 0.0366 & 0.5402 & 0.0001 \\
Crab eating macaque & 0.0339 & 0.5144 & 0.0001 \\
Squirrel monkey & 0.0434 & 0.4354 & 0.0001 \\
\hline
\end{tabular}

afor purifying selection $(\mathrm{dN}<\mathrm{dS})$. $\mathrm{dN}$, non-synonymous substitution; $\mathrm{dS}$, synonymous substitution.

consensus primer (forward, 5'-TATAGGAGCCTGGCTG AGGA-3' and reverse, 5'-CAGGCCTAGGTCCAGCAG-3'), designed based on previously reported, highly conserved primate MIOX sequences with the online UniPrime2 tool http://uniprime.batlab.eu (9). The complete MIOX CDS was amplified (based on the human MIOX gene sequence). The PCR thermal cycling program included initial denaturation at $94^{\circ} \mathrm{C}$ for $3 \mathrm{~min}$, followed by 28 cycles of denaturation at $94^{\circ} \mathrm{C}$ for $30 \mathrm{sec}$, annealing at $59^{\circ} \mathrm{C}$ for $35 \mathrm{sec}$, and elongation at $72^{\circ} \mathrm{C}$ for $45 \mathrm{sec}$, with a final elongation at $72^{\circ} \mathrm{C}$ for $6 \mathrm{~min}$. Universal $\beta$-actin gene primers (Ambion; Thermo Fisher Scientific, Inc.) were used to amplify the positive control. All PCR amplification products were 


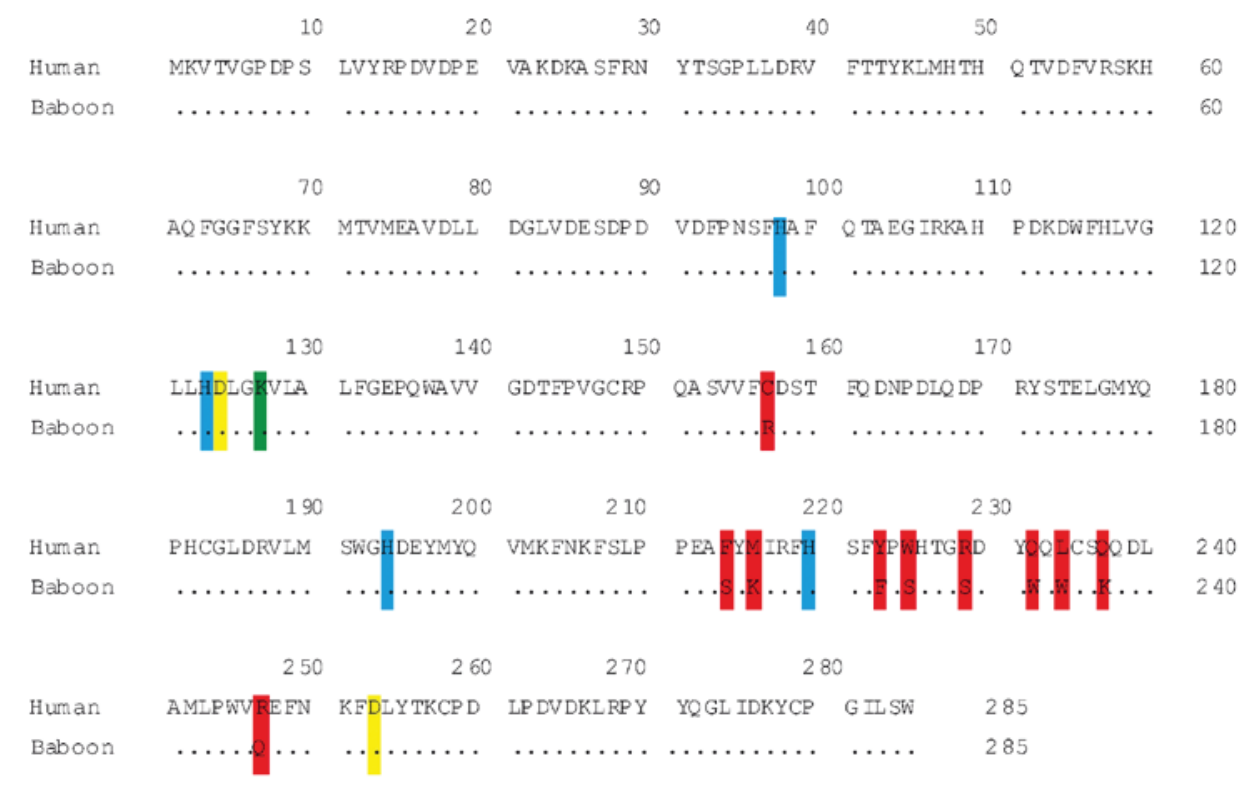

Figure 1. Alignment of human and baboon myo-inositol oxygenase protein. Structurally conserved amino acids are indicated in green (lysine, K127); blue for histidine (H98, H123, H194 and H220); and yellow for aspartate (D124 and D253). Ten amino acid differences are shown in red.

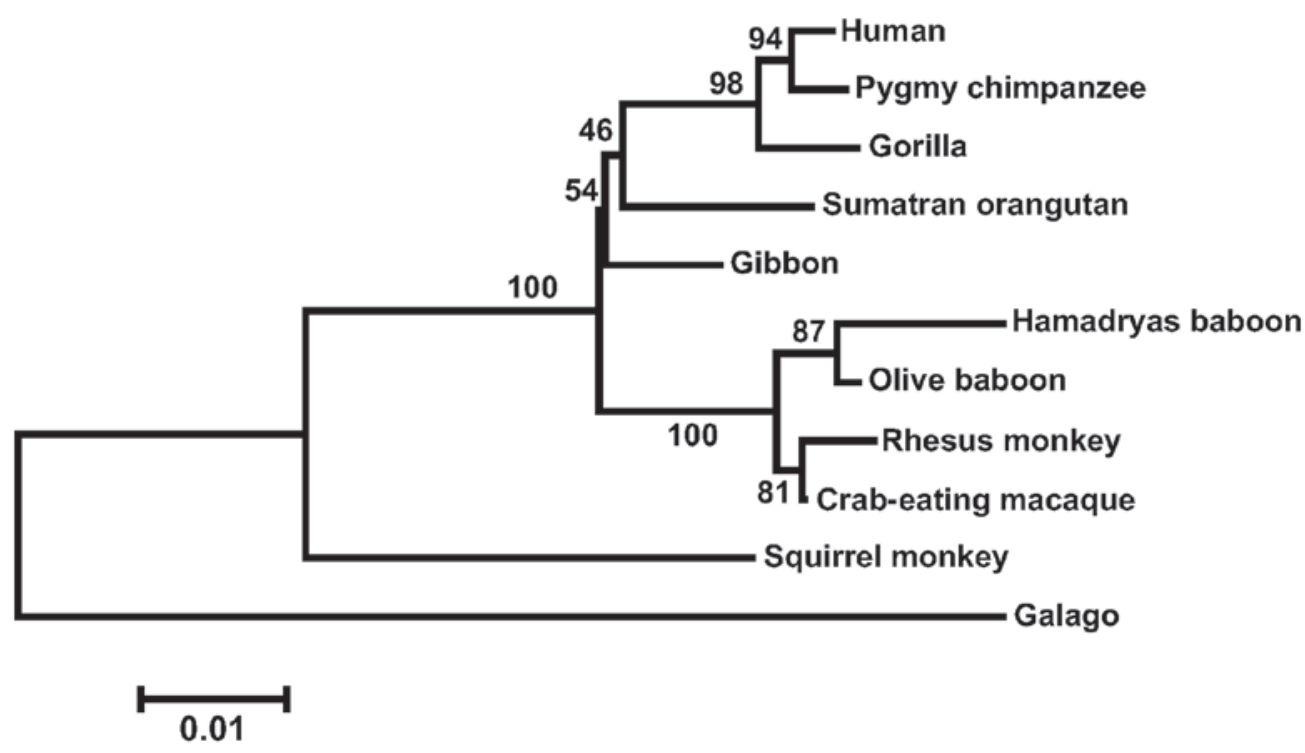

Figure 2. Phylogenetic tree of myo-inositol oxygenase coding sequences from various primates. The tree was constructed using MEGA version 6 with the neighbor-joining method and a bootstrap analysis with 1,000 replications. The bootstrap values are indicated on the branches. The lineage-specific clades represent the apes, Old World Monkeys, New World Monkeys and lemurs (out-group).

confirmed by electrophoresis in agarose gel (1\%) at $75 \mathrm{~V}$ for 35 min, stained with ethidium bromide and visualized under UV light. To visualize ethidium bromide-stained DNA gels we used a transilluminator (an ultraviolet lightbox).

Molecular cloning and sequencing. The PCR products were cloned with the TOPO ${ }^{\circledR}$ XL PCR Cloning kit with the $\mathrm{pCR}^{\circledR}$-XL-TOPO ${ }^{\circledR} 3.5 \mathrm{~kb}$ vector (Invitrogen; Thermo Fisher Scientific, Inc.). Electrocompetent Top 10 Escherichia coli cells (Invitrogen; Thermo Fisher Scientific, Inc.) were transformed with the ligation reactions, according to the manufacturer's instructions. The cloning products were sequenced with an ABI PRISM 3100 Genetic Analyzer (Applied Biosystems Life Technologies) using the universal
M13 primers and BigDye ${ }^{\circledR}$ Terminator Cycle Sequencing Reagents (Applied Biosystems; Thermo Fisher Scientific, Inc.). The electropherograms were analyzed with the GeneStudio Pro tool (GeneStudio, Inc., Suwanee, GA, USA). The DNA sequences of the MIOX transcripts were used to deduce the amino acid sequences using the Transeq online softwa http:// www.ebi.ac.uk/Tools/st/emboss_transeq/ (10), and these were subsequently aligned with other MIOX sequences using the ClustalW program (11). All procedures were performed with three clones of each amplicon to exclude artifacts.

Phylogenetic analysis. The data obtained from the sequencing analysis were used as the target sequence in a Basic Local Alignment Search Tool search to determine the identity. The 
sequences were subsequently aligned using the ClustalW program http://www.ebi.ac.uk/Tools/msa/clustalw2 (11). The GenBank accession numbers of the sequences used in the current study are presented in Table I. A phylogenetic tree was constructed from the mRNA sequences using MEGA version 6 software (12) with the neighbor-joining method (13) with 1,000 bootstrap replications (14). To identify the evolutionary forces underlying the divergence in the primate MIOX genes, the synonymous ( $\mathrm{dS}$; encoded amino acid does not change) and non-synonymous ( $\mathrm{dN}$; the encoded amino acid changes) substitutions were quantified using the modified Nei-Gojobori method (15), and the MIOX CDs from apes, Old World monkeys (OWMs) and New World monkeys (NWMs), with the lemur counterpart sequence as the out-group. The hypothesis of positive or adaptive evolution $(\mathrm{dN}>\mathrm{dS})$, purifying selection $(\mathrm{dN}<\mathrm{dS})$ and neutrality $(\mathrm{dN}=\mathrm{dS})$ was then tested using a codon-based $\mathrm{Z}$ test of selection (16), which is included in the MEGA version 6 software (12). $\mathrm{P}<0.05$ was considered to indicate a statistically significant difference.

\section{Results}

Molecular biology. A single band of the expected size (858 bp) was amplified by RT-PCR from renal tissue cDNA. This amplicon was not observed in the other tissue samples (testis, heart, omental fat, skeletal muscle, pancreas, mononuclear cells, liver or hypothalamus) that were analyzed. Thus, the MIOX transcript was only observed in the kidney. The novel sequence was deposited in the GenBank database (accession no. KP299165).

The nucleotide and amino acidic sequences of baboon MIOX were compared with their human counterparts. The percentage nucleotide similarity between the baboon and human genes was $95 \%$ and the percentage amino acid identity between the proteins was $96 \%$. In total, 10 amino acid changes were identified between the two primate species. Thorsell et al (1) proposed a novel role for Lys127 (K127) in the crystallized human MIOX protein in controlling access to the diiron cluster. That position is conserved in the baboon enzyme as predicted from the ORF. In our previous study, it was found that, in the active site of the baboon MIOX enzyme, the diiron cluster is bound in a conserved crevice formed between $\alpha 4, \alpha 5, \alpha 6, \alpha 7$ and $\alpha 8$ (17). This represents a strictly conserved diiron cluster with a first coordination sphere of four histidines (H98, H123, H194 and H220) and two aspartates (D124 and D253) (1). All these positions are conserved in the baboon MIOX protein (Fig. 1).

Phylogenetic analysis. Apes, OWMs and NWMs demonstrated a clear tendency toward purifying selection $(\mathrm{dN}<\mathrm{dS}$; $\mathrm{P}=0.0001$ ). A screening test was performed using Fisher's exact test selection with the models of Nei-Gojobori, Li-Wu-Luo, Pamilo-Bianchi-Li and Kumar. The same results were obtained with all the models used (Table II).

Statistical analysis. Statistical and mathematical calculations made in this work were performed using different algorithms of the MEGA software. Although there are many mathematical algorithms, none is better than another, they simply start from different assumptions. These algorithms are known and accepted among the expert community in the subject. The phylogenetic tree constructed from primate MIOX sequences (Fig. 2) shows four lineage-specific clades, corresponding to apes, OWMs, NWMs and lemurs (out-group). It supports the orthology within the primate MIOX genes. Bootstrap values are shown on the branches of the tree. Approaching results were obtained with other phylogenetic methods.

\section{Discussion}

Molecular biology. The MIOX sequence in baboons was observed to be highly similar to that of other apes. The preservation of exon-intron limits and the lack of evident mutations indicate that the baboon MIOX gene is functional. To the best of our knowledge, this is the first study that reports the expression profile of the novel MIOX gene in different tissues from baboons. The results of the current study indicate that, in baboons, the $M I O X$ gene is only expressed in the kidney, while in humans, MIOX gene expression is observed in kidney and extrarenal tissue (3). To elucidate this difference, further studies are required, which include promoter alignment, bioinformatics analyses (in order to predict transcriptional factor binding sites) and searching $\mathrm{CpG}$ islands that are susceptible to methylation. Conservation of the structural amino acid (lysine 127), histidines (98, 123, 194 and 220) and aspartates (124 and 253) (1), suggests that the three-dimensional shape may be identical in baboon and human MIOX protein (Fig. 1). Kidney expression in the two species suggests that the machinery, which regulates gene expression is similar in humans and baboons. In addition, it indicates that the MIOX gene may exert a similar physiological impact. However, further studies under different physiological conditions, such as insulin resistance, diabetes, obesity, fasting or other conditions associated with metabolic syndrome, are required.

Phylogenetic analysis. Positive selection $(\mathrm{dN}>\mathrm{dS})$ means that non-synonymous substitutions are functional and profitable to the organism, and provide an evolutionary benefit. While purifying selection $(\mathrm{dN}<\mathrm{dS})$ implies that evolutionary pressure eases off. The $\mathrm{dN}$ and $\mathrm{dS}$ rates were estimated in the present study and it was found that the evolutionary force that acts is purifying selection (Table II). Thus, it was hypothesized that purifying selection is an indication that the MIOX gene is functional in the studied species, as functional family genes fit this hypothesis. In conclusion, only one $M I O X$ gene was found for baboons and splicing variants were not detected. Furthermore, MIOX gene expression was only identified in the kidney. The $\mathrm{dN}$ and $\mathrm{dS}$ rates conclusively demonstrated that $M I O X$ genes are included in the hypothesis of purifying selection.

\section{Acknowledgements}

The present study was financed by grants from the Mexican Council of Science and Technology (CONACYT; grant nos. 167697 and 157965). The authors gratefully acknowledge the critical reading of the manuscript by Sergio Lozano-Rodríguez. 


\section{References}

1. Thorsell AG, Persson C, Voevodskaya N, Busam RD Hammarström M, Gräslund S, Gräslund A and Hallberg BM: Structural and biophysical characterization of human myo-inositol oxygenase. J Biol Chem 283: 15209-15216, 2008.

2. Arner RJ, Prabhu KS, Thompson JT, Hildenbrandt GR, Liken AD and Reddy CC: Myo-Inositol oxygenase: Molecular cloning and expression of a unique enzyme that oxidizes myo-inositol and D-chiro-inositol. Biochem J 360: 313-320, 2001.

3. Arner RJ, Prabhu KS, Krishnan V, Johnson MC and Reddy CC: Expression of myo-inositol oxygenase in tissues susceptible to diabetic complications. Biochem Biophys Res Commun 339: 816-820, 2006.

4. Arner RJ, Prabhu KS and Reddy CC: Molecular cloning, expression, and characterization of myo-inositol oxygenase from mouse, rat, and human kidney. Biochem Biophys Res Commun 324: 1386-1392, 2004.

5. Nayak B, Xie P, Akagi S, Yang Q, Sun L, Wada J, Thakur A, Danesh FR, Chugh SS and Kanwar YS: Modulation of renal-specific oxidoreductase/myo-inositol oxygenase by high-glucose ambience. Proc Natl Acad Sci USA 102: 17952-17957, 2005.

6. Yang B, Hodgkinson A, Millward BA and Demaine AG: Polymorphisms of myo-inositol oxygenase gene are associated with Type 1 diabetes mellitus. J Diabetes Complications 24: 404-408, 2010

7. Yang Q, Dixit B, Wada J, Tian Y, Wallner EI, Srivastva SK and Kanwar YS: Identification of a renal-specific oxido-reductase in newborn diabetic mice. Proc Natl Acad Sci USA 97: 9896-9901, 2000.

8. Zeng LC, Liu F, Zhang X, Zhu ZD, Wang ZQ, Han ZG and Ma WJ: hOLF44, a secreted glycoprotein with distinct expression pattern, belongs to an uncharacterized olfactomedin-like subfamily newly identified by phylogenetic analysis. FEBS Lett 571: 74-80, 2004.
9. Boutros R, Stokes N, Bekaert M and Teeling EC: UniPrime2: A web service providing easier Universal Primer design. Nucleic Acids Res 37: W209-W213, 2009.

10. Rice P, Longden I and Bleasby A: EMBOSS: The European Molecular Biology Open Software Suite. Trends Genet 16: 276-277, 2000.

11. Thompson JD, Gibson TJ, Plewniak F, Jeanmougin F and Higgins DG: The CLUSTAL_X windows interface: Flexible strategies for multiple sequence alignment aided by quality analysis tools. Nucleic Acids Res 25: 4876-4882, 1997.

12. Tamura K, Stecher G, Peterson D, Filipski A and Kumar S: MEGA6: Molecular Evolutionary Genetics Analysis version 6.0. Mol Biol Evol 30: 2725-2729, 2013.

13. Saitou N and Nei M: The neighbor-joining method: A new method for reconstructing phylogenetic trees. Mol Biol Evol 4: 406-425, 1987.

14. Efron B, Halloran E and Holmes S: Bootstrap confidence levels for phylogenetic trees. Proc Natl Acad Sci USA 93: 13429-13434, 1996.

15. Nei $M$ and Gojobori T: Simple methods for estimating the numbers of synonymous and nonsynonymous nucleotide substitutions. Mol Biol Evol 3: 418-426, 1986.

16. Zhang J, Rosenberg HF and Nei M: Positive Darwinian selection after gene duplication in primate ribonuclease genes. Proc Natl Acad Sci USA 95: 3708-3713, 1998.

17. Thorsell AG, Persson C, Voevodskaya N, Busam RD, Hammarström M, Gräslund S, Gräslund A and Hallberg BM: Structural and biophysical characterization of human myo-inositol oxygenase . J Biol Chem 283: 15209-15216, 2008. 